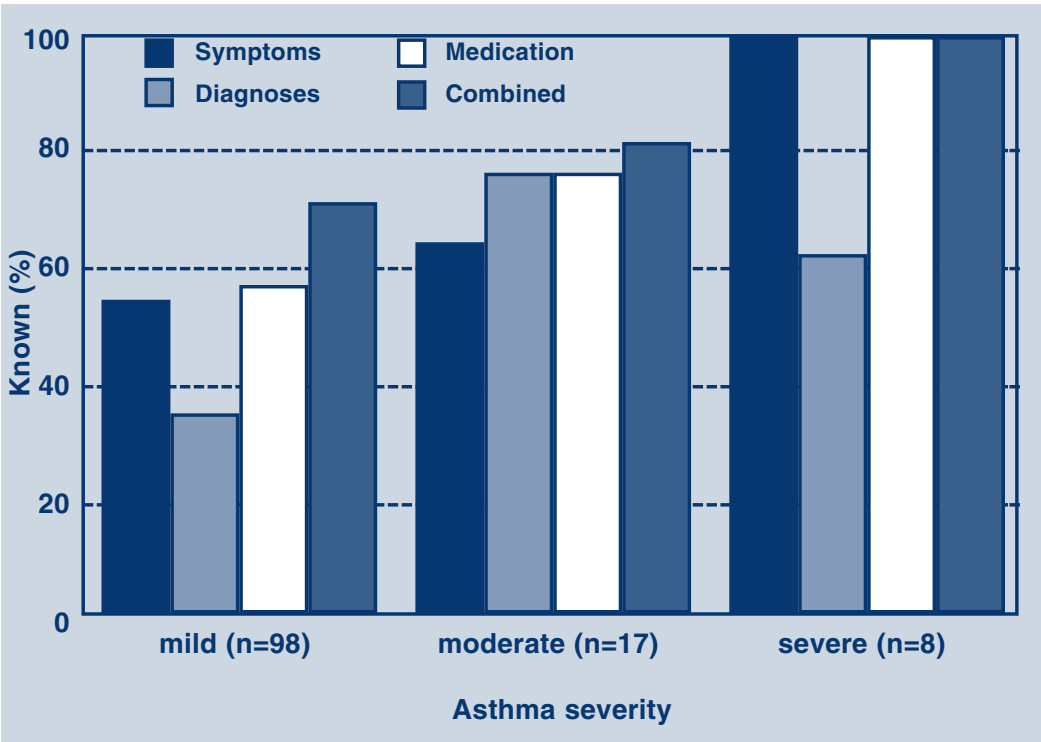

Figure 1: Proportion of asthmatic children known to their GP as indicated by registered symptoms, diagnoses and medication, shown according to degree of asthma severity international paediatric asthma consensus group. Arch Dis Child 1992; 67: $240-8$.

7. Kerrebijn KF. Consensus astma bij kinderen. Ned Tijdschr v Geneeskd 1993; 137: 1239-46.

8. McCarthy TP, Lenny W. Management of asthma in preschool children. Br J Gen Pract 1992; 42: 429-34.

9. Neville RG, Bryce FP, Robertson FM, et al. Diagnosis and treatment of asthma in children: usefulness of a review of medical records. Br J Gen Pract 1992; 42: 501-3.

10. Strachan DP. The prevalence and natural history of wheezing in early childhood. J R Coll Gen Pract 1985; 35: 182-4.

11. Luyt DK, Burton PR, Simpson H. Epidemiological study of wheeze, doctor-diagnosed asthma, and cough in preschool children in Leicestershire. BMJ 1993; 306: 1386-90.

12. Jones KP, Bain DJG, Middleton M, et al. Correlates of asthma morbidity in primary care. BMJ 1992; 304: 361-4.

13. Ferris BG. Epidemiology standardization project. Am Rev Respir Dis 1978; 118(suppl 6): 7-53.

14. Spelman R. Two year follow-up of the management of chronic or recurrent cough in children according to a asthma protocol. Br J Gen Pract 1991; 41: 406-9.

15. Rethans JJ. To what extent do clinical notes by general practitioners reflect actual medical performance? A study using simulated patients. Br J Gen Pract 1994; 44: 153-6.

16. Martinez FD, Wright AL, Taussig LM, et al. Asthma and wheezing in the first six years of life. N Engl J Med 1995; 332: 133-8. 17. Christie GL, Helms PJ, Ross SJ, et al. Outcome for children of parents with atopic asthma and transient childhood wheezy bronchitis. Thorax 1997; 52: 953-7.

18. Martin AJ, McLennan LA, Landau LI, et al. The natural history of childhood asthma to adult life. BMJ 1980; 280: 1397-400.

19. Dirksen WJ, Geyer RMM, de Haan M, et al. NHGstandaard astma bij kinderen. Huisarts Wet 1992; 35: 355-62.

\title{
Does implementing COPD guidelines improve patient care and save money in practice?
}

R.C.M. Jones and S. Copper

Rupert Jones

General Practitioner

\section{Shirley Copper}

Practice Nurse

The Roborough Surgery,

1 Eastcote Close,

Plymouth, Devon PL6 6HP, UK.

\section{Correspondence to:}

Dr R.C.M. Jones

Date received: 12/10/98

Date accepted: 31/01/99

Asthma in Gen Pract 1999; 7(1): 12-15

\section{ABSTRACT}

Objectives: To identify and assess the management of patients with COPD attending our practice asthma clinic by implementing protocols for the diagnosis and management of COPD, including reversibility testing.

Design and subjects: All patients aged over 39 years attending the asthma clinic at The Roborough Surgery were included. We assessed the implementation of the protocols and analysed prescribing data in those found to have irreversible airflow obstruction.

Results: COPD was found in 35/58 adults $(60 \%)$ over 40 years, of these, $6(17 \%)$ were irreversible. In irreversible patients, less inhaled steroids were prescribed, but this was offset by more anticholinergic prescriptions. The majority had had appropriate diagnostic tests, but the uptake of immunisation was $51 \%$ for influenza and $43 \%$ for pneumococcal infection.

Conclusion: Applying COPD protocols did not reduce prescribing costs, but encouraged optimum patient care in terms of investigations, diagnosis, appropriate treatment and immunisation.

\section{INTRODUCTION}

It has been stated that most patients with COPD have irreversible airway obstruction. ${ }^{1,2}$ These patients often receive expensive, but ineffective, drug treatment. ${ }^{3}$ As the disease progresses and they become more breathless, more treatment is added, with increased prescribing costs. Reversibility testing is useful in excluding chronic asthma from COPD and establishing whether drug therapy is likely to be beneficial. It has been predicted that large savings could be made if reversibility testing is systematically applied to patients with COPD in primary care. $^{3}$

In June 1996, the surgery introduced protocols for the diagnosis and management of COPD agreed by partners and nurses. These include reversibility testing, appropriate investigations, smoking advice, vaccination and treatment review. The protocols were produced in conjunction with British Thoracic Society (BTS) members, but preceded the publication of the BTS guidelines. ${ }^{1}$ They are compatible with the European Respiratory Society (ERS) guidelines. ${ }^{4}$ The primary aim of this audit was to establish how many 
patients attending the 'asthma' clinic had COPD, and to assess the use and impact of the management guidelines within our practice. The secondary aim was to assess the effect on prescribing costs of implementing a policy of stopping inhaled steroids in patients with irreversible airway obstruction.

\section{METHODS}

The study group consisted of all patients aged over 39 years who were attending the nurse-led clinic, which is supported by two general practitioners with an interest in respiratory disorders. The practice used two spirometers: the first, a small vane type (Medical Micro Ltd), was used for screening and in GP consultations. The second was a fully computerised type (Vitalograph 2120), which needed calibration whenever it was set up and was used by the nurse during asthma clinics. Being more accurate, it also was used to check the findings of the microspirometer.

The practice has one half-time and five full-time doctors caring for 10,700 patients [47\% (5030) aged over 39 years]. It is located in the northern suburbs of Plymouth and extends to the southern fringes of Dartmoor in southwest Britain. There is a wide range of social class with a deprivation index close to the national average.

\section{Protocols}

The protocols were designed in conjunction with Professor D. Shale of the Department of Respiratory Medicine at the University of Wales School of Medicine. The following were agreed by the primary care team.

All patients over 39 years of age attending the asthma clinic should have diagnosis by spirometry. If the $\mathrm{FEV}_{1}$ was more than $80 \%$ of expected or the $\mathrm{FEV}_{1}$ : FVC ratio was more than $75 \%$, COPD was excluded. Others had spirometry repeated and, if still low, underwent reversibility assessment. Those who could be reversed, by the methods listed below, to normal $\mathrm{FEV}_{1}$ or $\mathrm{FEV}_{1}$ : FVC ratio were diagnosed as asthma, the remainder with persistent airflow obstruction were deemed to have COPD.

\section{Reversibility assessment}

Reversibility was defined as a variation in $\mathrm{FEV}_{1}$ or peak expiratory flow (PEF) of more than $20 \%$. This was checked by the following methods:

1. Informal: Recent recordings of lung function in the patient's notes at times showed sufficient variability to confirm reversibility, for instance, before and after a course of prednisolone.

2. $\beta_{2}$-agonists: By using $5 \mathrm{mg}$ of salbutamol by nebuliser, acceptable alternatives include salbutamol, terbutaline or ipratropium bromide metered-dose inhaler via large volume spacer. Dramatic changes in $\mathrm{FEV}_{1}$ or PEF are suggestive of asthma rather than COPD and merits reconsideration of the diagnosis. The two conditions can coexist.

3. Inhaled corticosteroids: By using two to three months of high-dose inhaled corticosteroids (beclomethasone dipropionate or budesonide $800-1000 \mu \mathrm{g}$ or fluticasone propionate $500 \mu \mathrm{g}$ daily).

4. Oral steroids: Two weeks of prednisolone at a dose of $30 \mathrm{mg}$ daily.

\section{Chest X-ray}

A chest X-ray was recommended within two years of diagnosis and at least once every five years thereafter.

\section{Minimum data recording}

We agreed that the minimum data set would be recorded in all patients notes. Smoking habits were recorded as past exposure (best expressed as packyears) ${ }^{1}$ and current status. Smoking advice was recorded and leaflets were made available for patients to take home.

The presence of other pulmonary conditions was recorded, for example, tuberculosis and bronchiectasis as well as other significant medical conditions such as ischaemic heart disease, osteoporosis and diabetes.

\section{Vaccination status}

In our practice, we recommend influenza immunisation annually. We agreed that we would record whether patients were advised to have the vaccine, and if and when it was administered.

Pneumococcus immunisation was recommended to be administered on one occasion for all patients, according to current Department of Health advice.

\section{RESULTS}

Fifty-eight adults over 39 years of age attended the asthma clinic in the preceding 12 months. After spirometry according to the protocols, 35/58 (60\%) patients were diagnosed as having COPD. The mean FEV $_{1}$ was 1.31 , range $0.34-2.40$. Table 1 shows the grade of severity and results of reversibility testing in

Figure 1: Methods by which reversibility was confirmed in 20 patients, aged over 39 years, with COPD who were attending an asthma clinic in a general practice

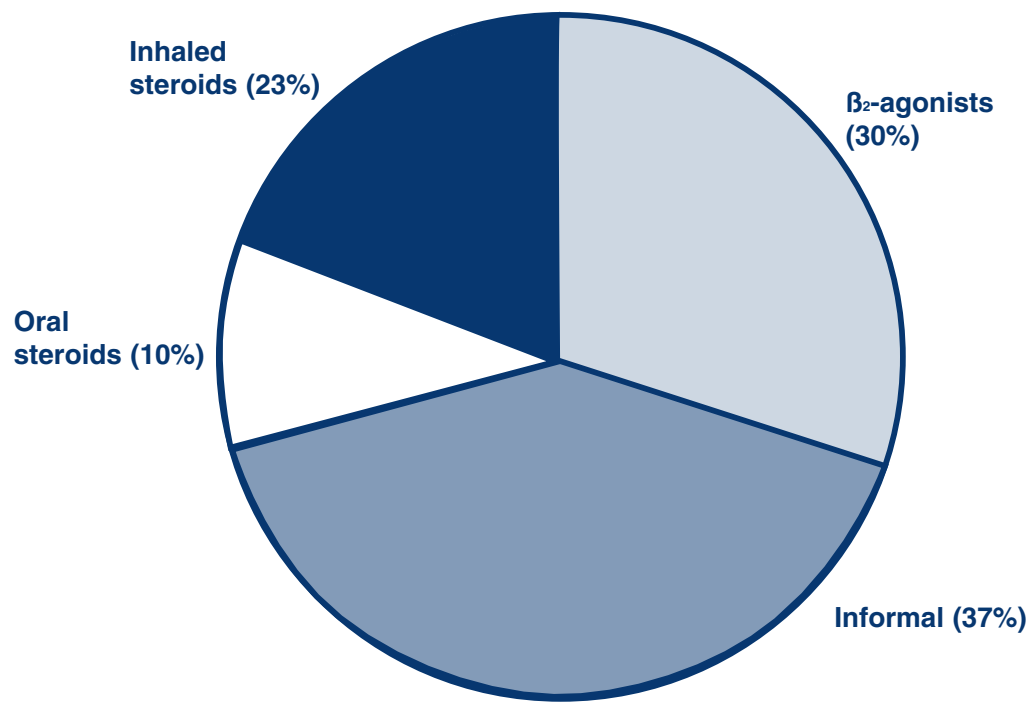


Table 1: Grade of severity of air flow obstruction and results of reversibility testing in $35 / 58$ patients with COPD attending the asthma clinic

\begin{tabular}{lcc} 
& Reversible & Irreversible \\
\hline All & 29 & 6 \\
Mild & 10 & 1 \\
Moderate & 11 & 3 \\
Severe & 8 & 2
\end{tabular}

Table 2: Changes in prescribing for six patients shown to be irreversible on formal testing. The figures represent total numbers of inhalers prescribed for these patients for six months before and six months after reversibility testing

\begin{tabular}{lcccc} 
& $\begin{array}{c}\text { Steroid } \\
\text { inhalers }\end{array}$ & $\begin{array}{c}\text { B2-agonist } \\
\text { inhalers }\end{array}$ & $\begin{array}{c}\text { Anticholinergic } \\
\text { inhalers }\end{array}$ & $\begin{array}{c}\text { Combination antichol/ } \\
\text { B2-agonist } \\
\text { inhalers }\end{array}$ \\
\hline $\begin{array}{l}\text { Before } \\
\text { After }\end{array}$ & 11 & 31 & 6 & 10 \\
$\begin{array}{l}\text { Change } \\
\text { in costs }\end{array}$ & $-£ 58.50$ & 31 & 13 & 15 \\
& & 0 & $+£ 29.40$ & $+£ 32.50$
\end{tabular}

those identified as having COPD. Reversibility was demonstrated in 29/35 (83\%) patients; the diagnosis was made most frequently by informal assessment from the records (Figure 1).

Prescribing data were analysed for six months before and after reversibility testing for those deemed to have irreversible obstruction (six patients; Table 2). Three patients with irreversible disease were on inhaled steroids - two stopped and one continued as his cough became worse after stopping. The reduction in the cost of inhaled steroids, however, was offset by the increase in anticholinergic prescriptions which were more likely to be supplied after attending the asthma clinic. Of 17 patients started on inhaled anticholinergic therapy, $70 \%$ are continuing long-term.

In the patients diagnosed as having COPD, a chest $\mathrm{X}$ ray was performed within two years of diagnosis in $57 \%$, within the last five years in $71 \%$, but five patients (14\%) had no chest X-ray at diagnosis or in the last five years. Immunisation for influenza within the last 12 months occurred in $51 \%$, and for pneumococcal infection within the last five years in $43 \%$.

Three of the 35 patients were taking B-blockers at the time of assessment; one was on aspirin and another on an oral non-steroidal anti-inflammatory agent.

\section{DISCUSSION}

This audit was limited to patients attending the asthma clinic and is not necessarily representative of all patients with COPD attending the surgery.

It is believed that airflow reduction in COPD is usually irreversible. ${ }^{1,2,4}$ However, data from the USA demonstrated that up to two-thirds of patients with COPD have a significant response to inhaled bronchodilators. ${ }^{6,7}$ Of our patients with COPD, 29 out of $35(83 \%)$ demonstrated evidence of reversibility. In practical terms, reversibility testing was easy: two-thirds could be assessed in minutes either via review of records or by response to nebulised bronchodilator. As in the case of the ERS statement, ${ }^{4}$ our guidelines originally did not include a minimum $200 \mathrm{ml}$ increase in $\mathrm{FEV}_{1}$, as recommended by the BTS guidelines, but were supported by (PEF) monitoring. We now suggest adherence to the BTS recommendation. As the PEF is less reliable, it may be misleading in COPD reversibility assessment especially in more severe cases, so there is an argument for the use of spirometry.

There are practical difficulties in stopping inhaled steroids in patients with irreversible COPD: They tend to have more severe disease and do not always take kindly to having their treatment reduced when they are getting worse. Even if there are no objective changes in spirometry, this does not necessarily mean that they are not benefiting from anti-inflammatory treatment. In such situations, it is difficult to blindly follow guidelines and stop inhaled steroids rather than listen to the patient. After all the guidelines still suggest using bronchodilators which may be equally ineffective.

As only a small minority of our patients with COPD was irreversible and had their prescriptions reduced, reversibility testing may not lead to marked reductions in prescribing costs in COPD. However, these results need to be considered cautiously as our numbers were small and may not be representative of other populations of COPD patients in primary care. The findings do justify a larger multicentre study; such a study is due to start shortly.

The process of systematic review of patients with COPD may, in fact, increase costs as other drugs may need to be added. Anticholinergic drugs have a particular place in $\mathrm{COPD}^{8,9}$ and we have advocated a trial of this therapy; $70 \%$ of those patients who started, continued to use these drugs. In this sample of patients, none were receiving regular oral steroids, theophyllines or long-acting bronchodilators.

Despite active encouragement, our influenza vaccination rates are unacceptably low, demonstrating the need for a register of those at risk to check that they are invited at the right time and that they attend. We also had low rates of uptake of pneumococcal immunisation, but this may be less important and is not routinely recommended by the British or European guidelines on COPD. ${ }^{1,4}$

The value of performing a chest X-ray in all cases is debatable. It is helpful to exclude other pathology, such as carcinoma of the bronchus. The X-rays in this group did not reveal any major pathology which required subsequent management. The BTS guidelines recommend chest X-rays in moderate-to-severe disease only, unless other symptoms are present. Previously, three patients in our practice had presented with cough, wheeze and a positive response to inhaled steroids, and 
subsequently proved to have bronchial carcinoma. Therefore, our guidelines err on the side of caution. In this group, it was encouraging that chest X-rays had been performed in the majority $(86 \%)$ albeit that not all were done at diagnosis or in the last five years.

B-blockers, aspirin and non-steroidal anti-inflammatory drugs are known to sometimes cause respiratory problems. Three patients attending the asthma clinic were being prescribed B-blockers; these were discontinued immediately with beneficial clinical effect. Iatrogenic causes of airway obstruction should not be overlooked; auditing patients helps to avoid such pitfalls.

\section{CONCLUSION}

Our study describes a method for diagnosis and management of patients with COPD in general practice. Use of COPD protocols in patients attending the asthma clinic optimised patient care in terms of investigations, diagnosis, appropriate treatment and immunisation. We were, however, unable to reduce our prescribing costs through inappropriate use of inhaled steroids in patients with irreversible airway obstruction. Further larger studies in this area are needed.

\section{References}

1. The British Thoracic Society, The National Asthma

Campaign, The Royal College of Physicians of London, et al.

Guidelines for the management of chronic obstructive

pulmonary disease. Thorax 1997; 52: S1-28.

2. Nisar M, Walshaw M, Earis JE, et al. Assessment of reversibility of airways obstruction in patients with chronic obstructive airways disease. Thorax 1990; 45: 190-4. 3. Peperell K, Rudolf M, Pearson M, et al. General practitioner prescribing habits in asthma/COPD. Asthma in Gen Pract 1997; 5: 29-30.

4. European Respiratory Society. Optimal assessment and management of chronic obstructive pulmonary disease. Eur Respir J 1995; 8: 1398-420.

5. Department of Health. Immunisation against infectious disease. Salisbury DM, Begg NT, eds. London, HMSO, 1996. 6. American Thoracic Society Statement. Standards for the diagnosis and care of patients with chronic obstructive airways disease. Am J Resp Crit Care Med 1993; 152: S77-120.

7. Goyal M, Caswell H, Cleland J. Diagnosis of asthma and chronic obstructive airways disease. Br J Gen Prac 1996; 47: $53-4$.

8. Tashkin DP, Ashutosh K, Bleeker ER, et al. Comparison of the anti-cholinergic inhaler ipratropium bromide with metaproterenol in chronic obstructive pulmonary disease: a multicentre study. Am J Med 1986; 81(Suppl 5a): 61-5. 9. Leitch AG, Hopkins JM, Ellis DA, et al. The effect of aerosol ipratropium bromide and salbutamol on exercise tolerance in chronic bronchitis. Thorax 1978; 33: 711-3.

\section{Letter to the Editor}

\section{Editor}

Dr Sheikh comments on the common association of rhinitis and asthma in his paper on 'Asthma and coexistent disease' (Asthma in Gen Pract 1998; 6(2): 15-18).

This was a personal problem for me, as I am an asthmatic. Effective treatment is to exhale through the nose after inhaling the preventive steroids prescribed for asthma. This is simple but seems to be little known.

\section{Dr G S Plaut}

Halstead, Essex, UK.
Editor,

Dr Plaut suggests that asking patients to exhale (via the nose) their inhaled asthma steroids is effective treatment for co-existent rhinitis. Whilst this sounds plausible, and may be of benefit to some patients, as far as I am aware, there is no published evidence supporting the routine use of such a strategy.

Highly effective, proven treatments for rhinitis, however, do exist and I would suggest that these are employed as first-line treatment options. ${ }^{2}$

Dr A Sheikh

Imperial College School of Medicine, London, UK.

1. Lund VJ, Aaronsen D, Bousquet J, et al. International consensus report on the diagnosis and management of rhinitis. Allergy 1994; 49(Suppl 19): 1-34.

2. Sheikh A. Rhinitis: fact sheet. GPIAG (in press).

Notes for contributors are available from The Editor, Asthma In General Practice, Strategic Medical Publishing Ltd, Action International House, Crabtree Office Village, Eversley Way, Egham, Surrey TW20 8RY, UK. 